\title{
А.Ю. Моисеева
}

\section{КВАЛИА-ФИЗИКАЛИЗМ ДЖ. ПЕРРИ КАК ОТВЕТ НА АРГУМЕНТ ЗНАНИЯ}

\begin{abstract}
Рассматривается предложенный Дж. Перри вариант защиты физикализма от аргумента знания, более известного как мысленный эксперимент «комната Мэри». Сочетая индексикальную стратегию объяснения результатов эксперимента с минимальнымм вариантом физикализма, этот философ показывает, что можно, не впадая в противоречие, принимать как тезис об эпистемической нередуцируемости квалиа, так и теорию тождества ментального и физического.

Ключевые слова: квалиа, физикализм, редукция, аргумент знания, комната Мэри.
\end{abstract}

Философия сознания в качестве самостоятельной дисциплины еще молода и не до конца концептуально оформилась, поэтому одной из важных задач для нее является переосмысление многих из тех понятий, которые применялись философами прошлого для описания сознания человека. Не являются исключением и такие понятия, как видимость, кажимость или, пользуясь старым философским термином, феномен. В процессе познания мы не можем избежать опоры на феномены, поскольку только через них мир становится эпистемически доступным для нас. Классическая философия уделяла много внимания вопросу о том, какие ограничения на нас накладывает такой способ познания и вправе ли мы вообще говорить, что способны познавать мир «внешних» объектов. Окончательный ответ на этот вопрос так и не был получен, однако сегодня большинство философов признает весьма вероятным, что, по крайней мере, наиболее успешные наши научные теории отражают объективные закономерности. Иначе трудно было бы объяснить, почему они работают, причем вне зависимости от того, кто их применяет. Современная философия сознания разворачивает фокус внимания с объектов на субъект и задается вопросом, способны ли мы объяснить с научной точки зрения, что такое феномены и какую роль они играют в познании, т.е. выразить предельные основания своих теорий языком самих этих теорий. До сих пор такая амбициозная задача ставилась только в рамках математики, однако ничто априори не противоречит тому, чтобы она могла быть поставлена в рамках естественных наук.

Однако с феноменами связано еще одно старое и весьма загадочное философское понятие, а именно квалиа. В современной философии под квалиа понимается феноменальный характер переживания некоторого состояния, имеющего место в конкретной психике в конкретный момент времени. (Более подробное определение см. в [10].) Предположительно, квалиа представляют собой нечто такое, что позволяет нам распознавать, запоминать и воспроизводить в памяти те или иные свои состояния по тому, как они переживаются. Мы знаем, каково это для нас - видеть свет, слышать гром или 
пробовать шоколад, быть грустным, радостным или напуганным, чувствовать опьянение или тошноту, находиться в депрессии или испытывать воодушевление. Именно поэтому о состояниях собственной психики мы обычно лучше осведомлены, чем о состояниях чужой (если, конечно, обращаем внимание на эти состояния и не имеем особых причин для самообмана по их поводу).

Наибольший интерес философов всегда вызывали сенсорные квалиа. Примечательно, что сами свои имена они заимствуют от свойств объектов внешнего мира. Да и другие квалиа при ближайшем рассмотрении выглядят так, как будто они отражают некоторые свойства объектов и событий во внешнем мире: мы, как правило, не чувствуем грусти, если не произошло что-то плохое, не чувствуем опьянения, если не пили перед этим алкоголь, и т.д. Логично предположить, что между квалиа и этими свойствами, по крайней мере, в норме имеется причинная связь. Но единственный вид причинности, известный нам до сих пор, - это физическая причинность, а квалиа, на первый взгляд, не являются физическими, что выглядит как проблема для теории. Можно физически описать механизмы реагирования нейронов на раздражители и обработки этой реакции в нервной системе, но это описание ничего не даст нам для понимания того, почему данная конкретная реакция должна сопровождаться данным конкретным субъективным переживанием или вообще каким-нибудь субъективным переживанием. Дж. Левин [7], впервые четко сформулировавший эту проблему, дал ей удачное название «объяснительный разрыв» (explanatory gap), под которым она с тех пор фигурирует в философских текстах.

Объяснительный разрыв ставит перед исследователем, стремящимся построить теорию психического, непростой выбор: нужно либо доказать, что квалиа, вопреки интуиции, могут быть объяснены в физических терминах, либо настаивать на существовании некоторых нефизических следствий физических причин. Первый путь обычно ведет к физикализму или функционализму, второй - к эпифеноменализму или интеракционному дуализму ${ }^{1}$. Однако как физикализм, так и функционализм представляются многим чересчур вульгарными, упускающими нечто существенное относительно квалиа, а именно их субъективно-качественный характер. Что же касается эпифеноменализма и интеракционного дуализма, то в контексте современной научной картины мира для большинства философов принятие тезиса о том, что в результате взаимодействия некоторых объектов реальности изменяются не только физические свойства этих объектов, но и некие дополнительные их свойства, непосредственно наблюдаемые только из субъективной перспективы, является весьма нежелательным. За три с лишним века, прошедших с начала дискуссии, мы нисколько не приблизились к консенсусу по поводу того,

\footnotetext{
${ }^{1}$ Есть также менее распространенные варианты. Так, вариантом монистической теории является панпсихизм, а вариантом дуалистической теории - неинтеракционный дуализм типа мальбраншевского. В панпсихизме квалиа не редуцируются к физическим свойствам, объявляются фундаментальными свойствами всей материи вообще. Соответственно, физическая и психическая причинность трактуются не как разные виды причинности, а как разные аспекты одной и той же причинности, для описания которой мы просто не имеем пока подходящих терминов. В неинтеракционном дуализме причинная связь между физическими свойствами и квалиа как таковая отрицается, а корреляция между ними объясняется действием внешней причины, например Бога. В настоящее время оба варианта являются экзотикой потому, что они предполагают крайне сильные и не подкрепляемые опытом допущения.
} 
редуцируемы ли квалиа к каким-то свойствам других типов, и если да, то к каким, зато изобрели массу новых аргументов в пользу и против каждой из точек зрения. Об одном из этих аргументов - так называемом аргументе знания - речь и пойдет в данной статье.

Оригинальной формулировкой аргумента знания был мысленный эксперимент, представленный Ф. Джексоном в статье «Эпифеноменальные квалиа» [6] и получивший широкую известность под названием «комната Мэри». Условия эксперимента таковы: Мэри - учёный, изучающий цвета, и она настолько преуспела, что знает все физические факты о цвете и его восприятии другими людьми, начиная с поведения, которое конкретный цвет может вызвать у человека, и вплоть до последовательностей нейрофизологических событий, которые регистрируются при восприятии цвета. Однако, согласно идее эксперимента, Мэри с самого рождения была заключена в комнате, окрашенной только в чёрно-белые цвета, и могла наблюдать внешний мир только через чёрно-белый монитор. Вопрос в том, узнает ли Мэри что-нибудь новое о красном цвете, когда ей позволят покинуть комнату и она увидит, например, спелый томат. Интуитивно кажется очевидным, что что-то узнает, а именно то, каково видеть красное. Но если это так, то квалиа нередуцируемы к физическим свойствам, поскольку все физические факты о красном цвете Мэри знала заранее.

Мысленный эксперимент Ф. Джексона - одна из наиболее удачных попыток показать, что объяснительный разрыв действительно существует и является проблемой для редуктивных теорий квалиа. Значение эксперимента трактуется философами в очень широком диапазоне. Некоторые авторы полагают, что наличие разрыва в объяснении означает его наличие и в мире, т.е. физическое описание мира неполно не только эпистемологически, но и онтологически. Эта точка зрения наиболее полно выражена Д. Чалмерсом в ходе его аргументации в пользу эпифеноменализма (см. [2, 4].) В дискуссии о квалиа она занимает антиредукционистский полюс. На противоположном полюсе находится, например, каузальный функционализм Д. Деннета. (По поводу квалиа см. [5].) Умеренная позиция представлена нередуктивным физикализмом, который у каждого философа имеет свои особенности (см., например, [1]). Следует признать, что с точки зрения здравого смысла умеренная позиция по вопросу о природе квалиа представляется предпочтительной, однако в большинстве своем нередуктивные физикалистские теории не доходят до объяснения того, почему квалиа не могут быть редуцированы к физическим свойствам, если они в действительности являются физическими.

На этом фоне выгодно отличается стратегия, которая получила в литературе название индексикальной. Эта стратегия не только объясняет, почему новое знание Мэри является действительно новым, она еще и позволяет сформулировать такую концепцию знания, в которой случай Мэри не является чем-то исключительным. Именно этой стратегии придерживается Дж. Перри, развивая свою теорию, которую называют квалиа-физикализмом, поскольку она предполагает, что, с одной стороны, квалиа являются физическими свойствами, с другой стороны, знания о квалиа невыводимы из знаний о физических свойствах. С точки зрения квалиа-физикализма всякое знание включает в себя, помимо объектного, также рефлексивный компонент, кото- 
рый определяет, как данное знание должно применяться в некоторой ситуации. Именно различием в этом рефлексивном компоненте объясняется тот факт, что Мэри, зная все о физических свойствах переживания красного, не может вывести из своего знания феноменальные свойства этого переживания.

В книге «Знание, возможность и сознание» (Knowledge, Possibility and Consciousness, 2001) Дж. Перри начинает раскрывать свое видение проблемы объяснительного разрыва с того, что устанавливает различие между собственно феноменальными свойствами и нашими понятиями об этих свойствах. Как он пишет [8. С. 48], феноменальные свойства существенно отличаются от других свойств и отношений тем, что само их наличие означает их эпистемическую доступность. Например, чтобы узнать, что Берлин - большой город, мы должны или сами побывать в нем и оценить его величину, или узнать этот факт из книг, карт, со слов других людей и т.п. Чтобы узнать, каково наше переживание красного, нам не нужно удовлетворение каких-то дополнительных условий - в эпистемическом смысле это переживание всегда «с нами», коль скоро оно имеется у нас. Но из этого не следует, что всякий раз, когда мы видим красный, мы фактически знаем об этом. Чтобы узнать, нам нужно, во-первых, обратить внимание на данное переживание, во-вторых, обладать понятием, позволяющим отличать те переживания, которые являются переживаниями красного, от тех, которые ими не являются.

Здесь следует подчеркнуть, что понятие красного цвета как такового не тождественно понятию переживания красного цвета. В норме между ними существуют определенные когнитивные отношения. Так, если в какой-то момент субъект, скажем, убежден, что имеет переживание красного, то это убеждение обычно является причиной того, что он убежден, что в зоне его видимости находится нечто красное. Однако даже эти отношения не универсальны. Например, человек, страдающий цветовыми галлюцинациями и знающий об этом, может сомневаться, действительно ли видимый им объект красный, не сомневаясь при этом, что он действительно имеет переживание красного. Особенность таких случаев в том, что понятие феноменального свойства не выполняет должным образом ту адаптивную функцию, которую оно выполняет в норме, т. е. не является достойным доверия индикатором соответствующего реального свойства. Именно это заставляет нас считать галлюцинации нежелательными. Люди (за исключением, возможно, некоторых философов) убеждены, что их феноменальный опыт является реальностью, проецирующейся на реальность «внешнего» мира, в частности, на реальность их деятельности в этом мире. Они убеждены, что от того, как они действуют в определенных обстоятельствах, зависит то, какой опыт они получат в результате. Например, человек, впервые ударивший собственный палец молотком, как правило, немедленно приходит к выводу, что чтобы избегать в будущем переживаний, имеющих то же неприятное феноменальное свойство, нужно избегать бить молотком по пальцам. Способность рационально прийти к такому выводу зиждется на том, что, во-первых, имеется понятие феноменального свойства, во-вторых, имеется понятие реального события, в-третьих, имеется убеждение, в котором оба этих понятия ассоциированы посредством понятия причинного отношения, а именно убеждение в том, что первое переживание, имеющее это феноменальное свой- 
ство, было вызвано не чем иным, как данным событием - ударом молотка по пальцу. Таким образом, понятия феноменальных свойств играют важную роль в практическом рассуждении, но только если мы убеждены в их связи с реальностью.

Метафорически понятие вообще можно представлять себе в виде папки с документами, содержащими записи, полученные из различных источников. Папка поименована именем объекта, свойства или отношения, понятие о которых она содержит. Объект может быть связан с папкой различными способами. Во-первых, он может быть причиной создания папки. Во-вторых, он может быть причиной создания большинства записей в папке. В-третьих, об объекте может быть истинна большая часть записей в папке. В-четвертых, объект может быть тем, в отношении чего будет использоваться большая часть записей в папке. Со свойством дело обстоит аналогичным образом. Дж. Перри пишет: «Наши понятия о свойствах могут содержать всевозможные вещи: частные объекты, которые имеют свойство, типичные причины наличия свойства у объектов, типичные эффекты его наличия, критерии распознавания проявлений свойства, изображения в памяти объектов, имеющих свойство, имена свойства и т. д. Какие вещи мы найдем, будет зависеть от того, какие свойства, которыми обладают концепции, а также от того, как они вовлечены в нашу собственную жизнь, - и ниже - В случае понятия о феноменальном опыте кажется, что его основная часть, по крайней мере, во многих случаях должна быть связана с тем, каков этот опыт был и каков он будет для нас, обладателей понятия» [8. С. 153-154]. Эту демонстративнораспознавательную часть понятия, формирующуюся в процессе переживания данного опыта, Перри называет юмовской идеей. Именно в этой части существует сходство между вспоминаемым, предвосхищаемым и непосредственно переживаемым опытом, которое позволяет нам учиться на собственных ошибках. Так, человек из предыдущего примера, вспомнив, насколько неприятным было ощущение удара молотком по пальцу, сможет предсказать, что ощущение удара молотком по голове тоже будет неприятным настолько, что лучше и не пробовать.

Конечно, кроме демонстративно-распознавательной части, понятие о феноменальном свойстве содержит и описательную часть: типичные причины этого свойства, его типичные эффекты и т.д. Благодаря этой описательной части мы можем судить о переживаниях других людей на основании воспоминаний о наших собственных переживаниях в сходных обстоятельствах, поскольку все мы (опять же, за исключением некоторых философов) убеждены в том, что не только наш опыт, но и опыт других людей проецируется на реальность «внешнего» мира. Например, мы имеем основания полагать, что наш собеседник чувствует боль, если видели, как он только что ударил молотком себе по пальцу, и сами имели опыт боли от удара по своему пальцу молотком. И мы имеем еще большие основания полагать так, если он при этом стонет, поскольку и мы обычно стонем, когда чувствуем боль. Как полагает Дж. Перри, наиболее обоснованные суждения о переживаниях других людей мы можем получить, исходя из данных нейронауки.

Таким образом, переживание можно в принципе описать с помощью только функциональных или только физических фактов. Вопрос о том, может 
ли полученное описание быть таким, чтобы ему удовлетворяло одно и только одно феноменальное свойство (скажем, феноменальное свойство переживания красного), на данном этапе не поддается окончательному разрешению. Но даже если и может, это описание будет лишь косвенным образом указывать на интересующее нас свойство. Единственным способом прямо (демонстративно) указать на феноменальное свойство является указание на опыт, обладающий этим свойством. По этому поводу Дж. Перри пишет: «Сила термина "субъективный", как я его использую, не в том, чтобы отрицать возможность для других быть осведомленными о событиях, происходящих в [психике] другого человека, а в том, чтобы утверждать, что существуют особые методы, доступные для субъекта. Есть такие методы познания опыта, которые могут быть применены только тогда, когда у субъекта имеется этот опыт, и только этим субъектом, и которые являются прямыми, по крайней мере, в смысле отсутствия участия органов и средств внешнего восприятия» [8. С. 57].

В отсутствие опыта, обладающего искомым феноменальным свойством, мы можем знать кое-что об этом свойстве на основании описаний чужого опыта, но такое знание будет существенно неполным. Пользуясь словом из обыденного языка, можно сказать, что в этом случае мы можем сформировать представление, но такое представление не будет юмовской идеей. Пока мы не получим опыт, мы не узнаем, соответствует ли наше представление действительности, даже если оно точно ей соответствует. Суть различия между юмовской идеей и обычным представлением состоит в том, что последнее не обладает правильным типом причинной зависимости от опыта. Если воспользоваться аналогией, то можно сказать, что обычное представление похоже на фотографию одного из близнецов, которую используют, чтобы опознать другого близнеца. Сходство может быть абсолютным, но это не дает нам права говорить, что это его фотография. Точно так же мы не имеем права говорить, что мы знаем, каково феноменальное свойство некоторого опыта, если по факту наше знание получено без участия самого этого опыта.

С точки зрения обыденной интуиции утверждение, что феноменальные термины прямо указывают на опыт, а не на его функциональную роль или физическую реализацию, кажется очевидно истинным. Можно отрицать, что оно истинно, как делают некоторые современные философы. Но, как подчеркивает Дж. Перри [8. С. 79], принятие его за истину не обязывает к тому, чтобы перестать быть физикалистом. Следует различать физикализм максимальный, утверждающий, что феноменальные термины не могут указывать ни на что кроме физических свойств, и физикализм минимальный или, по выражению Перри, антецедентный, утверждающий, что феноменальные термины могут указывать на физические свойства. Этот последний допускает, что феноменальные термины указывают на нередуцируемо феноменальные свойства, но считает правдоподобным, что все феноменальные свойства в конечном счете тождественны каким-то физическим свойствам. Теория, которой придерживается обсуждаемый нами автор, принадлежит ко второму типу и совмещает тезис о нередуцируемости квалиа с тезисом о тождестве ментального и физического. За это ее и называют квалиа-физикализмом. 
По мнению Дж. Перри, вся защита антецедентного физикализма против аргумента знания сводится к тому, чтобы показать, как можно обладать двумя независимыми понятиями об одном и том же феноменальном свойстве. Свою мысль он поясняет сначала на примере понятий об объектах. В семантике существует старая проблема, состоящая в том, что истинностное значение предложений, говорящих нечто о пропозициональных установках субъекта (его убеждениях, желаниях, надеждах и т.д.) по отношению к некоторому объекту, зависит от того, как этот объект именуется. Все выглядит так, как будто в контекстах с пропозициональными установками нарушается принцип подстановочности тождественного. Поиск решения для данной проблемы привел Г. Фреге к тому, чтобы признать, что в таких контекстах значениями имен становятся не референты, а понятия, которые субъект связывает с этими именами. Однако его решение было небезупречным. Позже предлагались и другие решения, более или менее удачные, но каждое из них вызывало серьезную критику. Некоторые философы дошли до того, что объявили предложения с предикатами пропозициональных установок вообще не выражающими содержание этих установок, а только описывающими их. Однако, как полагает Перри, мы должны не выбрасывать за борт содержание таких предложений, а как можно больше узнать о нем. Именно это он и пытается сделать в своей книге.

Если излагать семантические взгляды Дж. Перри кратко, то они состоят в следующем. Содержанием, согласно его точке зрения [8. С. 125], называется некоторый способ классификации языковых и когнитивных репрезентаций по их условиям истинности или, в более общем виде, условиям выполнимости. Действие этого способа классификации Перри поясняет с помощью специальной формулы - Анализатора содержания (Content Analyzer), - которая выглядит так:

(0) Если дано $B, \varphi$ истинно, если и только если $A$, где $\varphi$ означает репрезентацию, имеющую значение истинности, $B$ - некоторые фиксированные фоновые условия, при которых оценивается истинность $\varphi$, а $A$ - содержание, приписываемое $\varphi$, если даны эти фоновые условия ${ }^{1}$. Иначе говоря, $A$ есть условия, которые должны выполняться дополнительно к условиям $B$, чтобы $\varphi$ было истинно. Ясно, что, если мы варьируем $B$, варьируется и $A$. Согласно Перри, полное содержание репрезентации определяется полными условиями ее истинности $C$ такими, что для всех возможных сочетаний $A$ и $B$ в формуле (0) верно, что $A \in C$ и $B \in C$.

Чтобы проиллюстрировать, как работает Анализатор содержания, Дж. Перри использует пример, придуманный К. Донелланом для иллюстрации различения атрибутивного и референциального употребления определенных дескрипций, а именно предложение «Убийца Смита безумен». Аналогично различению Донеллана, Перри различает два способа прочтения этого предложения. Первый способ фиксирует в левой части формулы (0) ответы на вопросы о том, на каком языке высказано предложение, каковы семантические и синтаксические правила данного языка, применяемые для

${ }^{1}$ Следует сказать, что в этой работе Дж. Перри не использует слово «фон» для обозначения тех условий, которые принимаются как данность, но оно встречается в других его работах (см.: [9. С. 200, $210,266])$ и, на наш взгляд, хорошо выражает суть дела. 
интерпретации предложения, а также кто является референтом имени «Смит», и не фиксирует денотат дескрипции «убийца Смита»:

(1) Если дано, что $\chi$ есть предложение, в котором выражение «убийца Смита» означает единственного убийцу Смита, то $\chi$ истинно, если и только если существует единственный убийца Смита, и он безумен.

Но предположим, что нам дано, что убийцей Смита является Джонс. Тогда появляется второй способ прочтения, состоящий в том, чтобы ввести новое условие в левую часть формулы $(0)$ :

(2) Если дано, что $\chi$ есть предложение, в котором выражение «убийца Смита» указывает на Джонса, то $\chi$ истинно, если и только если Джонс безумен.

Согласно Дж. Перри, ни один из этих двух способов не дает единственно правильного ответа на вопрос об объектном содержании (subject matter content) предложения «Убийца Смита безумен», т.е. о том, что данное предложение сообщает о мире. В каждом отдельном случае произнесения предложения решить, какое содержание передается, атрибутивное или референцииальное, можно только на основании экстралингвистических фактов. Поэтому правильный ответ должен состоять в том, что оба этих содержания являются компонентами объектного содержания, и какой из компонентов будет релевантным в конкретном случае, зависит от контекста. Как подчеркивает Перри, содержание наших языковых и когнитивных репрезентаций не является чем-то данным свыше. Именно мы приписываем его, сообразуясь с теми знаниями о языке и о мире, которые могут быть релевантны в данном контексте.

Существуют и другие способы приписать репрезентации содержание. Один из них состоит в том, чтобы поставить само индивидуирующее свойство в зависимость от контекста. Парадигмальный случай такой зависимости употребление личных местоимений. Для примера рассмотрим следующую ситуацию. Пусть Смит имеет убеждение, которое выражает предложением «Джонс убийца», и приобрел он это убеждение без личного знакомства с Джонсом. Пусть, далее, однажды Смит лицом к лицу встречается с Джонсом и дружелюбно беседует с ним, не зная, что это и есть Джонс, но в какойто момент вдруг слышит, как некто окликает его собеседника «Эй, Джонс!». Вполне естественно ожидать, что его поведение по отношению к собеседнику после этого момента изменится: Смит начнет проявлять нервозность, постарается поскорее ретироваться или станет незаметно искать подходящее средство самозащиты. Теперь зададимся вопросом: приобрел ли Смит новое убеждение, которое могло бы служить объяснением этих перемен? Очевидно, что, если бы Джонс спросил его об этом и если бы Смит пожелал ответить искренне, он сказал бы «Я приобрел убеждение, что ты - убийца». Проинтерпретировав с использованием Анализатора содержания придаточное предложение его высказывания (для краткости обозначим это предложение $\psi$ ), получим два варианта:

(3) Если дано, что $\psi$ есть предложение, в котором местоимение «ты» указывает на Джонса, то $\psi$ истинно, если и только если Джонс убийца. 
(4) Если дано, что $\psi$ есть предложение, в котором местоимение «ты» о3начает адресата этого предложения, то $\psi$ истинно, если и только если адресат $\psi$ убийца.

В (3) дополнительные условия истинности задают референциальное (оно же в данном случае и объектное) содержание аналогично тому, как это было в (2) выше. Содержание, которое задано в (4), называется у Дж. Перри рефрлексивным содержанием (reflexive content) и представляет собой то, что данное предложение сообщает относительно ситуации его произнесения. Можно было бы подумать, что рассматривать рефлексивное содержание отдельно от объектного содержания не нужно, так как первое сводимо ко второму. Однако сам Перри [8. 68] считает, что эта точка зрения, которую он обозначает как предположение объектности (subject matter assumption), в корне неверна. Несводимость рефлексивного содержания проявляется, по его мнению, всегда, когда в предложении нас интересует не его истинность, а то, что мы можем из него заключить о ментальных состояниях и поведении субъекта, который его произносит.

Действительно, в нашем примере объектное содержание предложения $\psi$ ничем не отличается от объектного содержания предложения «Джонс убийца», которым Смит выражал свое старое убеждение. Если бы убеждения определялись только объектным содержанием, нельзя было бы сказать, что Смит приобрел новое убеждение и, следовательно, нельзя было бы объяснить перемены в его поведении. Но рефлексивное содержание предложения $\psi$ не совпадает с рефлексивным содержанием предложения «Джонс убийца», поскольку рефлексивное содержание этого последнего описывается условием, чтобы человек, которого говорящий называет Джонсом, был убийцей, и в этом условии ничего не говорится об адресате. Таким образом, именно рефлексивное содержание отличает новое убеждение Смита от старого и позволяет объяснить поведение Смита. Более того, оно позволяет предполагать, что кто бы ни произнес искренне «Ты - убийца» и каков бы ни был его адресат, он с высокой вероятностью будет проявлять нервозность, стараться ретироваться или искать средство самозащиты. Иначе говоря, поведение во многих случаях зависит от рефлексивного содержания и не зависит от объектного содержания. Отсюда можно заключить, что для целей психологии и семантики контекстов с пропозициональными установками рефлексивное содержание является более удобным способом классификации языковых и когнитивных репрезентаций. ${ }^{1}$

Ситуацию с Мэри и переживанием красного Дж. Перри объясняет аналогично тому, как мы только что объяснили ситуацию со Смитом и Джонсом. Он рассматривает три аспекта знания, имеющегося у Мэри, когда она покинула черно-белую комнату и увидела спелый томат. Во-первых, некоторое имеющееся у нее знание можно было бы выразить предложением «Это переживание - переживание красного». Обозначим данное предложение $\xi_{1}$ и сформулируем его рефлексивное содержание:

(5) Если дано, что $\xi_{1}$ есть предложение, выражающее знание некоторого субъекта, и что выражение «это переживание» означает переживание, на ко-

\footnotetext{
${ }^{1}$ Подобную идею Дж. Перри впервые высказал в [9. С. 65].
} 
торое направлено внимание этого субъекта в момент, когда он имеет знание, выражающееся в $\xi_{1}$, то $\xi_{1}$ истинно, если и только если переживание, на которое направлено внимание субъекта в тот момент, является переживанием красного.

Заметим, что определить переживание, которое Мэри получит, выйдя из комнаты и увидев спелый томат, как переживание красного она могла бы, еще находясь в комнате. Несомненно, она знала заранее, что спелые томаты красны. Но она не могла «указать» на это переживание, поскольку оно просто отсутствовало в тот момент. Она могла лишь описать его.

Во-вторых, кое-что о переживании красного Мэри знала уже давно благодаря представлению о нем, основанному на теоретических знаниях, полученных, пока она находилась в комнате. Введем обозначение $Q_{R}$ для этого ее знания. Тогда знание, которое она имела до выхода из комнаты и продолжала иметь после выхода, мы сможем выразить в предложении « $Q_{R}-$ феноменальное свойство переживания красного». Обозначим полученное предложение $\xi_{2}$ и посмотрим на его содержание:

(6) Если дано, что $\xi_{2}$ есть предложение, выражающее знание некоторого субъекта, то $\xi_{2}$ истинно, если и только если понятие о $Q_{R}$, вовлеченное в данное знание, удовлетворяется переживанием красного.

В-третьих, из конъюнкции $\xi_{1}$ и $\xi_{2}$ можно вывести предложение « $Q_{R}-$ феноменальное свойство этого переживания». Это предложение и будет выражать новое знание, которое Мэри приобрела о $Q_{R}$. Обозначим его как $\xi_{3}$. Рефлексивным содержанием $\xi_{3}$ будет

(7) Если дано, что $\xi_{3}$ есть предложение, выражающее знание некоторого субъекта, и что выражение «это переживание» означает переживание, на которое направлено внимание этого субъекта в момент, когда он имеет знание, выражающееся в $\xi_{3}$, то $\xi_{3}$ истинно, если и только если переживание, на которое направлено внимание субъекта в тот момент, удовлетворяет понятию о $Q_{R}$, вовлеченному в данное знание.

Таким образом, выйдя из комнаты, Мэри дополнила свое понятие о $Q_{R}$ юмовской идеей, то есть образцом для распознавания сходных переживаний в будущем. Теперь, если она встретит незнакомый красный объект, ей не нужно будет анализировать отражающие свойства его поверхности, чтобы понять, что этот объект красный. Она может просто сравнить наличное переживание с тем переживанием красного, которое она имела до этого, и понять, что они принадлежат к одному типу.

Как и в случае со Смитом и Джонсом, изменение в знаниях Мэри не объясняется изменением в объектном содержании ее репрезентаций. В самом деле, зафиксировав референты выражений «это переживание» в $\xi_{3}$ и «переживание красного» в $\xi_{2}$, мы получим одно и то же содержание этих предложений. Но если абстрагироваться от референтов, то содержание будет разным. При этом мы вовсе не обязаны говорить, что новое знание Мэри полностью выражается в (7). Мы можем сказать, что тем, что она узнала, является необходимая истина, что $Q_{R}=Q_{R}$, и будем правы, поскольку именно таково объектное содержание $\xi_{3}$. Однако сказав так, мы не объясним, почему это знание является новым. Чтобы получить адекватное объяснение, мы должны использовать что-то вроде (7). 
Далее Дж. Перри переходит к тому, чтобы показать, что физикализм в его минимальном варианте не противоречит признанию нередуцируемости квалиа. Он пишет [8. С. 166], что точка зрения, согласно которой существуют способы познания физических фактов, субъективные в том простом и естественном смысле, который был описан ранее, противоречит не физикализму, а предположению объектности. Это предположение гласит, что существует вид знания, который состоит в усмотрении истинности некоторой языковой или когнитивной репрезентаций без какой-либо субъективной перспективы, «взглядом из ниоткуда». Однако нет никаких существенных причин принимать такое предположение. На самом деле существование знания, лишенного рефлексивного содержания, крайне сомнительно. Даже если у нас имеется некое сугубо аналитическое знание типа «Всякий безумный убийца - убийца», мы все же связываем его с некими понятиями о безумии и убийцах, предназначенными для использования в конкретных жизненных ситуациях и, следовательно, содержащими ссылку на какую-то субъективную перспективу.

Таким образом, Дж. Перри удается защитить физикализм от аргумента знания путем сугубо эпистемологической интерпретации последнего. Против такой интерпретации тоже, конечно, выдвигаются некоторые доводы. В частности, некоторые сторонники онтологической интерпретации аргумента знания используют для атаки на минимальных физикалистов, подобных Перри, средства семантики возможных миров (см., например, [11].) Они говорят, что теория тождества ментального и физического, если представить ее утверждения в семантике возможных миров, предполагает, что все миры, в которых имеет место ментальное событие, но не имеет места соответствующее физическое событие, невозможны. Но знание в той же семантике возможных миров моделируется как функция, разделяющая все множество возможных миров на два непустых подмножества: миры, совместимые с данным знанием, и миры несовместимые с ним. Если нет ни одного возможного мира, совместимого со старым знанием Мэри, но несовместимого с ее новым знанием, то и самого этого нового знания нет.

На подобные возражения можно ответить, что они скрыто опираются на предположение объектности, ограничивая все доступное Мэри знание лишь знанием объектных фактов. Дж. Перри же настаивает на том, что существует особый тип фактов - рефлексивные факты [8. С. 159]. Один и тот же объектный факт в разных субъективных перспективах соответствует разным рефлексивным фактам, и знание нового рефлексивного факта является новым знанием, даже если никакой новый объектный факт при этом не познается. Если бы мы сами не считали, что возможно новое знание старого объектного факта, невозможно было бы объяснить, почему мы считаем естественным изменение поведения Смита по отношению к Джонсу и почему мы считаем естественным удивление Мэри при виде первого в ее жизни спелого томата, увиденного без посредства черно-белого монитора. Все это означает, что когда мы оцениваем модальные характеристики некоторого положения дел по отношению к некоторому знанию, нужно учитывать не только объектные факты, составляющие это положение дел, но и все рефлексивные факты, которые могут в него входить. 
Для большей ясности попытаемся выразить идею Дж. Перри языком семантики возможных миров. Что значит утверждение, что Мэри знает, каково видеть красный цвет? С точки зрения семантики возможных миров это значит, что она знает, как описать переживание, которое она имела бы при виде чего-то красного, таким образом, что во всех возможных, исходя из данного его знания, мирах, это переживание и только оно удовлетворяло бы описанию. Так вот, используемое здесь понятие возможности, исходя из некоторого знания, можно трактовать двояко. Можно понимать возможность как реальную возможность. Тогда при условии, что описание истинно и полно, исходя из его знания, Мэри следует считать возможными лишь те миры, где переживание красного имеет место тогда и только тогда, когда описание удовлетворяется. С другой стороны, можно понимать возможность как конизептуальную возможность. Тогда, даже исходя из знания Мэри истинного и полного описания, некоторые миры, где в определенный момент времени переживание красного есть, хотя описание не удовлетворено, или, наоборот, переживания красного нет, хотя описание удовлетворено, следует считать возможными. Конечно, эти миры не будут реально возможными при условии, что описание истинно и полно. Однако, как нам представляется, применительно к данному случаю нужно использовать понятие концептуальной возможности, поскольку понятие реальной возможности означает, что Мэри обладает не только истинным и полным описанием, но и знанием того, что описание истинно и полно. Однако сам факт, что описание истинно и полно, не является физическим фактом о восприятии красного цвета, он является фактом об этом описании. Данный факт выходит за пределы знания, которое приписывается Мэри по условиям эксперимента, и не следует логически из этого знания. Поэтому без изменения условий мы не имеем права утверждать, что ей известен этот факт.

Отвергнув предположение объектности, мы ничуть не погрешим против физикализма, если скажем, что миры, совместимые со старым знанием Мэри, но не совместимые с ее новым знанием, лишь реально невозможны, но концептуально возможны для нее - или, пользуясь формулировками Дж. Перри, они невозможны исходя из объектного содержания знания Мэри, но возможны исходя из рефлексивного содержания ее знания. Точно так же можно сказать, что возможность того, что Джонс находится в здравом уме, существует исходя из атрибутивного содержания знаний некоторого детектива, которому известно, что убийца Смита безумен, но не существует исходя из референциального содержания его знаний. Таким образом, вместо одного множества возможных миров мы должны получить иерархию таких множеств, отображающуюся в иерархию всех содержаний, которые можно приписать данному знанию субъекта. Следует признать, однако, что стандартное понятие возможного мира противится такому использованию, поскольку мир рассматривается как максимальное непротиворечивое описание некоторого объектного (но не обязательно реально существующего) положения дел. «Материалом» для построения миров являются те же самые объекты и свойства, которые составляют реальный мир, хотя в некоторых возможных мирах они будут присутствовать в других комбинациях, а некоторые объекты и свойства - во- 
все отсутствовать ${ }^{1}$. Поэтому для всякого объекта или свойства возможного мира, для которого в этом мире истинно утверждение о его тождестве какому-то другому объекту или свойству, то же самое утверждение будет истинно и в любом другом возможном мире. Видимо, семантика возможных миров просто не годится для моделирования содержания пропозициональных установок ${ }^{2}$.

\section{Лuтература}

1. Серл Дж. Открывая сознание заново. М.: Идея-Пресс, 2002.

2. Чалмерс Д. Сознающий ум: В поисках фундаментальной теории. М.: Либроком, 2013.

3. Barwise J., Perry J. Situations And Attitudes // The Journal of Philosophy. 1981. V. 78, № 11. P. 668-691.

4. Chalmers D. Phenomenal Concepts and the Explanatory Gap // Phenomenal Concepts and Phenomenal Knowledge: New Essays on Consciousness and Physicalism / T. Alter and W. Walter (eds.). NY: Oxford University Press, 2005. P. 167-194.

5. Dennet D.C. Sweet Dreams: Philosophical Obstacles to a Science of Consciousness. MA: MIT Press, 2005. P. 122-150.

6. Jackson F. Epiphenomenal Qualia. // The Philosophical Quarterly. 1982. Vol. 32, № 127. P. 127-136.

7. Levine J. Materialism and Qualia: The Explanatory Gap // Pacific Philosophical Quarterly. 1983. № 64. P. 354-361.

8. Perry J. Knowledge, Possibility and Consciousness. Cambridge, MA: MIT Press, 2001.

9. Perry J. The Problem of the Essential Indexical and Other Essays. NY, Oxford: Oxford University Press, 1993.

10. Tye M. Qualia // The Stanford Encyclopedia of Philosophy (Winter 2016 Edition). Edward N. Zalta (ed.) [Электронный pecypc]: https://plato.stanford.edu/archives/win2016/entries/qualia/ (дата обращения: 15.05.2017).

11. Nida-Rümelin M. Qualia: The Knowledge Argument. // The Stanford Encyclopedia of Philosophy (Summer 2015 Edition). Edward N. Zalta (ed.) [Электронный pecypc]: https://plato.stanford.edu/archives/sum2015/entries/qualia-knowledge/ (дата обращения: 16.05.2017).

Anna Yu. Moiseeva. Institute of Philosophy and Law of the Siberian Branch of the RAS (Novosibirsk, Russian Federation)

E-mail: abyssian03@gmail.com

DOI: $10.17223 / 1998863 X / 40 / 7$

\section{ARGUMENT \\ J. PERRY'S QUALIA-PHISICALISM AS AN ANSWER TO THE KNOWLEDGE}

Key words: qualia, phisicalism, reduction, the knowledge argument, Mary's room

The article is dedicated to one of the most complicated questions of philosophy of mind - reducibility of phenomenal properties or qualia to properties of other types. There are a lot of arguments used by proponents of irreducible qualia to prove their position. Likely, the knowledge argument, better known as "Mary's room" thought experiment, is the most discussed of them. It's a story about girl who has been imprisoned in black and white room since her birth, where she, impulsed by her curving to unknown, has studied every single physical fact about color, and then she was released to see the colored world with her own eyes. Intuition tells that Mary, who has leaved the room, will study something new about colors, for example, what it is like to see red. It's usually considered that accept-

\footnotetext{
${ }^{1}$ Или, более строго, некоторые объекты не будут существовать, а некоторые свойства не будут представлены ни в одном из существующих объектов.

${ }^{2}$ В нескольких местах своей книги [8. С.122, 193, 202] Дж. Перри намекает, что для анализа пропозициональных установок могло бы подойти понятие частично описанного возможного мира или ситуации. Семантика, в которой вместо возможных миров используются ситуации, развивается им в нескольких работах (см., например: [9. С. 130-137, 153-191]; а также совместно с Дж. Барвайзом [3].) Однако действительно ли с помощью ситуационной семантики можно ясно и интуитивно адекватно моделировать содержание знаний и убеждений - это вопрос для отдельного исследования.
} 
ing the intuition testifies against physicalism about qualia. John Perry, the author of "Knowledge, Possibility and Consciousness" (2001), disagrees with this position. Basing on analysis of examples from semantics of propositional attitudes, Perry formulates his own concept of content, assuming that any knowledge contains a reflexive component that bounds the subject matter component of this knowledge to some subjective perspective - a perspective, from which this knowledge was received or from which it can be used in practice. As he shows further, the novelty of Mary's knowledge of colors is in that reflexive component. So, assuming existence of some special non-physical properties is not necessary to accept intuitive result of thought experiment with Mary, it is sufficient to distinguish subjective perspectives.

\section{References}

1. Searle, J. (2002) Otkryvaya soznanie zanovo [A Re-Discovery of the Mind]. Translated from English by A.F. Gryaznov. Moscow: Ideya-Press.

2. Chalmers, D. (2013) Soznayushchiy um: V poiskakh fundamental'noy teorii [The Conscious Mind: In Search of a Fundamental Theory]. Translated from English by V. Vasiliev. Moscow: Librokom.

3. Barwise, J. \& Perry, J. (1981) Situations and Attitudes. The Journal of Philosophy. 78(11). pp. 668-691. DOI: $10.2307 / 2026578$

4. Chalmers, D. (2005) Phenomenal Concepts and the Explanatory Gap. In: Alter, T. \& Walter, W. (eds) Phenomenal Concepts and Phenomenal Knowledge: New Essays on Consciousness and Physicalism. New York: Oxford University Press. pp. 167-194.

5. Dennet, D.C. (2005) Sweet Dreams: Philosophical Obstacles to a Science of Consciousness. MA: MIT Press. pp. $122-150$.

6. Jackson, F. (1982) Epiphenomenal Qualia. The Philosophical Quarterly. 32(127). pp. 127136. DOI: $10.2307 / 2960077$

7. Levine, J. (1983) Materialism and Qualia: The Explanatory Gap. Pacific Philosophical Quarterly. 64. pp. 354-361. DOI: 10.1111/j.1468-0114.1983.tb00207.x

8. Perry, J. (2001) Knowledge, Possibility and Consciousness. Cambridge, MA: MIT Press.

9. Perry, J. (1993) The Problem of the Essential Indexical and Other Essays. New York, Oxford: Oxford University Press.

10. Tye, M. (2016) Qualia. In: Zalta, E.N. (ed.) The Stanford Encyclopedia of Philosophy. Winter 2016 Edition. [Online] Available from: https://plato.stanford.edu/archives/win2016/entries/qualia/. (Accessed: 15th May 2017).

11. Nida-Rümelin, M. (2015) Qualia: The Knowledge Argument. In: Zalta, E.N. (ed.) The Stanford Encyclopedia of Philosophy. Winter 2016 Edition. [Online] Available from: https://plato.stanford.edu/archives/sum2015/entries/qualia-knowledge/. (Accessed: 16th May 2017). 